\title{
Load Balancing of Peer-to-Peer MMORPG Systems with Hierarchical Area-of-Interest Management
}

\author{
Satoshi Fujita ${ }^{1}$ \\ ${ }^{1}$ Department of Information Engineering, Hiroshima University \\ Higashi-Hiroshima, 739-8527, Japan \\ E-mail: fujita@se.hiroshima-u.ac.jp
}

\begin{abstract}
This paper studies the load balancing problem in distributed systems designed for massively multiplayer online role-playing games (MMORPGs). More concretely, we consider a distributed system of masterworker type in which each worker is associated with a particular region in the game field, and propose a scheme to balance the load of workers as much as possible. The basic idea of the proposed scheme is to dynamically adjust the number of workers associated with each region according to the number of players in the region. The performance of the scheme is evaluated by simulation. The simulation results indicate that it reduces the response time of a previous scheme proposed by $\mathrm{Yu}$ et al. by about $50 \%$, which varies depending on the mobility pattern of the players.
\end{abstract}

Keywords: Load balancing, master-worker model, MMORPG, area-of-interest.

\section{Introduction}

In recent years, online video games such as Final Fantasy $\mathrm{XI}^{*}$ and EverQuest ${ }^{\dagger}$ have attracted many game users. For example, it is reported that the number of active users of Final Fantasy XI reaches one million in 2014 These MMORPGs (massively multiplayer online role-playing games) easily cause a bottleneck at the servers since in these games, a huge number of players interact with each other within a virtual space in realtime. To overcome such a crucial issue, MMORPGs supported by the Peer-to-Peer (P2P) technology have been proposed in the literature ${ }^{1,3,5,6,11}$. In this paper, we focus on the management of the information on players

*http://www.playonline.com/ff11/index.shtml

${ }^{\dagger}$ https://www.everquest.com/

\#http://www.hd.square-enix.com/eng/pdf/ar_2014_01en.pdf in such P2P MMORPGs. In particular, we consider the distributed execution of the area-of-interest management ${ }^{2,8}$ (AOIM, of short) in P2P environments.

AOIM is a technique to reduce the management cost of the game server by restricting the field of vision of each player (see Figure 1 for illustration). Such a restricted vision field is referred to as the AOI of the player and it generally has a "circular" shape centered at the player. The goal of AOIM is to continuously, selectively notifies game events occurred in the AOI to each player (examples of game events include the movement in the field and the conversation with other players). Since players move as the game proceeds, the system should trace the position 
of all players to update their AOI, i.e., the set of players contained in the AOI, as well as the tracking of game events related to these players. In order to realize such a management task in a P2P environment with no centralized control, we need to carefully design the way of notifying such events to the players, i.e., who and when notifies the events to the players.

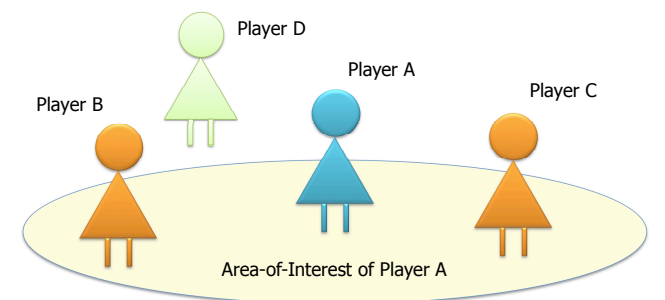

Fig. 1. Area-of-interest of Player A (Players B and C are visible from Player A, but Player D is not).

In this paper, we propose a scheme to realize a quick update of the AOI of players participating in P2P MMORPGs. Our scheme is an improvement of the scheme called MOPAR proposed by $\mathrm{Yu}$ et al. ${ }^{10}$. As will be described later, MOPAR statically partitions the given game field into several subfields called cells and delegates a part of the management task to a peer selected from peers associated to the cell. The selected peer is called the master of the cell, and updates the AOI of players in the cell through the communication with masters managing adjacent cells. With such an approach, we could effectively delegate a part of the task of the server to the participants. However, in the original protocol proposed by Yu et al., the number of masters in each cell is fixed to (at most) one, which causes a heavy load if many players concentrate in a specific cell. Such a heavy load of the master increases the response time of the updates, which degrades the comfortability of the resulting game environment. The basic idea of our scheme is to dynamically adjust the number of masters in each cell using a thresholdbased merge-and-split mechanism. Here, in conducting a splitting, we need to avoid a discontinuous change of the AOI such that many players suddenly appears or disappears. In the following, we propose several techniques to resolve such issues. The performance of the proposed scheme is evaluated by simulation. The simulation results indicate that the proposed scheme reduces the response time of the previous scheme by about $50 \%$, which varies depending on the mobility pattern of the players.

The remainder of this paper is organized as follows. Section 2 describes preliminaries. Section 3 describes the proposed scheme. Section 4 shows the simulation results. Finally, Section 5 concludes the paper with future work.

\section{Preliminaries}

\subsection{Model of P2P System}

Consider a distributed system consisting of a central server $S$ and a set of peers $P$, where each peer in $P$ is identified with a player in the game field. The server $S$ plays the following two roles:

- Authentication server which securely manages the charging information such as: 1) the purchase of items and 2) the skill and the level of each player.

- Management server which maintains the set of peers $P$ with their attributes. It does not keep the dynamic information such as game events and the position of players in the game field.

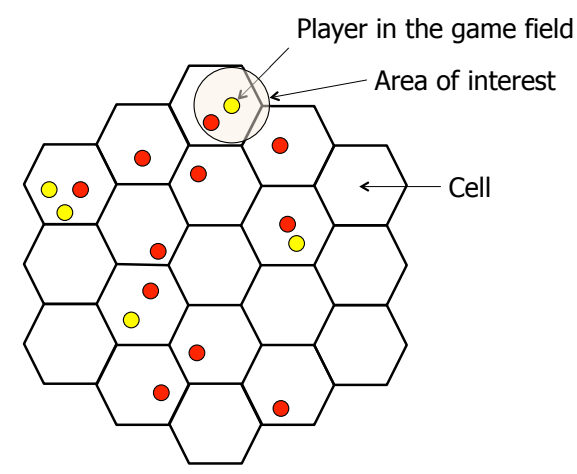

Fig. 2. Game field partitioned into hexagonal cells (yellow circle means a player corresponding to an ordinary peer and red circle means a player corresponding to a master).

The game field is partitioned into hexagonal cells as shown in Figure 2, where the size of cells can be independent of the AOI of players. Let $\mathscr{R}=\left\{R_{1}, R_{2}\right.$, $\left.\ldots, R_{n}\right\}$ be the resulting set of cells. At each point in time, each player in $P$ exists in a cell in $\mathscr{R}$. Let $P_{i}$ be a variable representing the set of peers existing in $R_{i}$. 


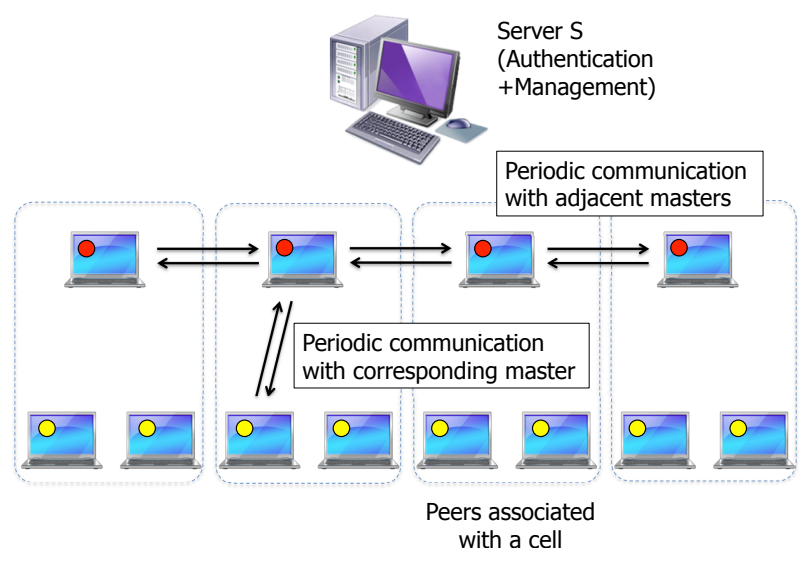

Fig. 3. Communication among peers in the $\mathrm{P} 2 \mathrm{P}$ model used in MOPAR (peers with red circle represent masters).

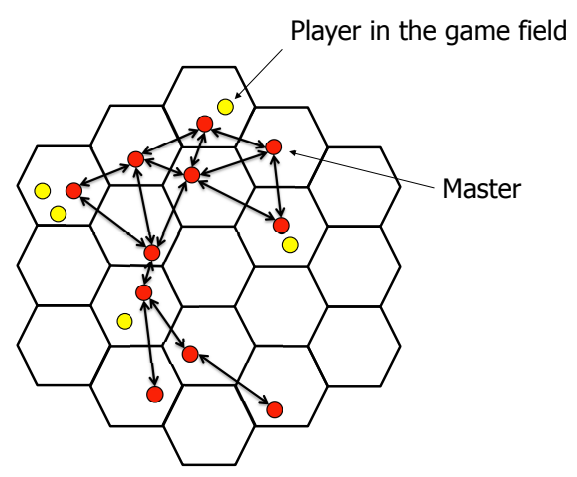

Fig. 4. Communication with masters of adjacent cells.

\subsection{Management of Dynamic Information}

For each $i$ with $P_{i} \neq \emptyset$, the server $S$ selects a peer in $P_{i}$ according to an appropriate rule and promotes it as the master of cell $R_{i}$.

The master of cell $R_{i}$ maintains the information on all peers existing in $R_{i}$, such as ID, current position in $R_{i}$ and IP address, where the position of each player is periodically collected to the corresponding master. The collected information is selectively notified to the relevant peers. More concretely, upon detecting that a player $v$ enters the AOI of player $u$ existing in $R_{i}$, the master of $R_{i}$ notifies the fact to $u$ with the IP address of $v$ so that $u$ can start the direct communication with $v$ which will (automatically) terminate when the counterpart of communication leaves the AOI. See Figure 3 for illustration. The change of AOI of players is detected by communicating with the masters of adjacent cells; i.e., it could be accurately acquired even if the AOI of a peer intersects with more than one cell as shown in Figure 2. Figure 4 shows the communication between adjacent masters. Note that we can bound the number of adjacent masters to be communicated by three, if the size of cells is sufficiently large compared with the size of AOI.

In MMORPGs, each player walks around the game field to meet other players or to clear specific missions. This causes a frequent crossing of the boundary of adjacent cells. To correctly reflect such a behavior of players to the set of masters, in MOPAR $^{10}$, player $u$ conducts the following operations before leaving cell $R_{i}$

- If $u$ is the master of $R_{i}$, it selects a random peer $v$ in $P_{i}-\{u\}$ and hands over the role of master to $v$ (such a selection is not conducted if $\left|P_{i}\right|=1$ ).

- Otherwise, it simply removes the information on $u$ from the master of $R_{i}$.

On the other hand, it conducts the following operations before entering cell $R_{j}$

- If $P_{j} \neq \emptyset$, then $u$ enters $R_{j}$ by sending its ID and IP address to the master of $R_{j}$.

- Otherwise, it simply becomes the master of $R_{j}$.

Note that the master of each cell can be identified by referring to the server $S$ (in MOPAR, such a behavior is realized by using the lookup to the Pastry ${ }^{9}$ ).

\subsection{Model of Peers}

Peers are modeled as follows (for simplicity, we assume that the server $S$ has a sufficiently high capability so that it can complete any computation and communication within a unit time). In a unit time, each peer executes the following sequence of operations:

- Receive messages from the input buffer;

- Conduct necessary computation; and

- Send messages to other peers.

Sent messages are delivered to the buffer of receivers within a constant time; i.e., we omit the effect of message routing and congestion avoidance. Each 
receiver receives a constant number of messages in a unit time, while the size of buffer is not limited to avoid overflow. Unreceived messages should be processed in the next cycle.

\section{Proposed Method}

\subsection{Classification of Game Events}

At first, we classify game events into three types:

1. Type-A: Events which occur at a specific point in the field, e.g., shopping at a market and boss fight at a specific place (e.g., dungeon or bridge).

2. Type-B: Interaction with other players, e.g., conversation and fight, which can start after receiving the IP address on the corresponding peer.

3. Type-C: Change of AOI, e.g., the entering and the leave of a player.

The objective of the proposed scheme is to support Type-C events, where in the following, we assume that Type-A events are handled by the server $S$ and Type-B events are realized by the local communication between peers. The reader should note that the support of Type-C events is crucial to realize an attractive online video game since the precise recognition of AOI is a key issue to conduct a timely interaction with nearby players.

\subsection{Load Balancing Scheme}

In MOPAR, the number of messages handled by the master of cell $R_{i}$ is proportional to $\left|P_{i}\right|$ and the load of the master is proportional to $\left|P_{i}\right|^{2}$ since it needs to calculate the distance for all pairs of the players. Under the model of P2P systems described in Section 2 , such a load of masters directly affects the update time of AOI. The key idea of the proposed scheme is to dynamically adjust the number of peers managed by each master. More concretely, we associate several peers (masters) to each cell and each master associated with cell $R_{i}$ manages a subset of $P_{i}$.

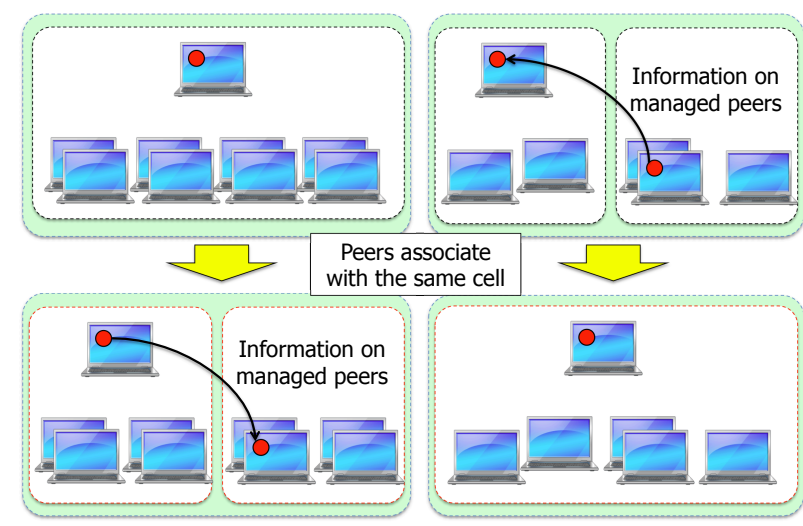

(a) Split of subset

(b) Merge of subsets

Fig. 5. Split and merge operations.

Let $U(u)$ denote the set of peers managed by master $u$. Set $U(u)$ dynamically changes by conducting split and merge operations triggered by two thresholds $T_{U}$ and $T_{L}$. More concretely,

- if $|U(u)|>T_{U}, u$ equally partitions $U(u)$ into two subsets and hands over one subset to a new master $v$ which is randomly selected from $U(u)$ (see Figure 5 (a) for illustration).

- if $|U(u)|<T_{L}$, then after identifying other master $v$ associated with the same cell $R_{i}$ (if any), $u$ asks $v$ to merge $U(u)$ and $U(v)$ into a single set (see Figure 5 (b) for illustration).

If $|U(u) \cap U(v)|>T_{U}$, then the resulting set is split into two halves again, so that the number of peers managed by each master is always within the range of $\left[T_{L}, T_{U}\right]$. In order to avoid unnecessary fluctuation, we fix two thresholds to satisfy relation $2 T_{L}<$ $T_{U}$, e.g., $T_{U}=3 \times T_{L}$. Recall that the list of masters is maintained by the server $S$ and is updated appropriately.

By applying split and merge operations, the number of "observable" peers in the AOI changes as follows?

- A split reduces the number of peers to a half, while it is still large enough since the size after the split is at least $T_{L}$.

- The number of peers increases by a merge, but it is not too large since the size of the set after the merge (and a subsequent split) is at most $T_{U}$.

\footnotetext{
$\S$ The reader should note that once the IP address of a peer such as the partner in a party is known, it can communicate with the peer even after being disappeared from the AOI due to split operation.
} 


\subsection{Splitting Algorithms}

This paper proposes three splitting algorithms. The first algorithm splits the given subset $U(u)$ by the peer ID, the second algorithm splits $U(u)$ in a random manner and the third algorithm splits $U(u)$ so that pairs of peers which have recently interacted belong to the same subset as much as possible.

\subsubsection{ID-based splitting}

In the first algorithm, which will be referred to as the ID-based splitting, the master $u$ simply divides $U(u)$ into two halves after sorting it in a non-decreasing order of the peer ID. If peer IDs are given in the order of participation, it naturally realizes a situation in which two peers which start the game almost at the same time will be visible with each other even when the game field becomes congested.

\subsubsection{Randomized Splitting}

In the second algorithm, the master $u$ randomly selects $|U(u)| / 2$ peers from set $U(u)$ and separates them as a new subset. In other words, for each peer in $U(u)$, a half of peers in the AOI disappear after splitting, independent of the participation order and the frequency of interaction.

\subsubsection{Cut-Based Splitting}

The third algorithm is based on the bipartition of a player interaction graph (PIG, for short) representing the interaction between players. PIG for the master $u$ has vertex set $U(u)$ and an edge set $E(u)$ so that two vertices are connected by an edge if and only if their corresponding players have interacted within the past $\tau$ time units (note that $u$ can construct such a graph by collecting all game events from players in $U(u)$ ). The graph bipartition problem is the problem of partitioning the vertex set so that the size of each subset is (almost) equal and the number of edges connecting two subsets is the smallest. Although the graph bipartition problem is NP-hard, there are several efficient local search algorithms such as Kernighan-Lin (KL) algorithm ${ }^{4}$ which solve the problem in a heuristic manner. In the proposed scheme, we use the KL algorithm to realize a split of $U(u)$ in a heuristic manner.

\subsection{Efficient Communication with Adjacent Cells}

In MOPAR, the master of cell $R_{i}$ periodically exchanges the position of players in $P_{i}$ with masters of adjacent cells so that the AOI of each player is correctly updated. In the proposed scheme, we associate several masters for each cell and the number of masters associated with $R_{i}$ increases as the number of players in $P_{i}$ increases. This means that we need to take care of the tradeoff between traffic and the freshness of the update of AOI.

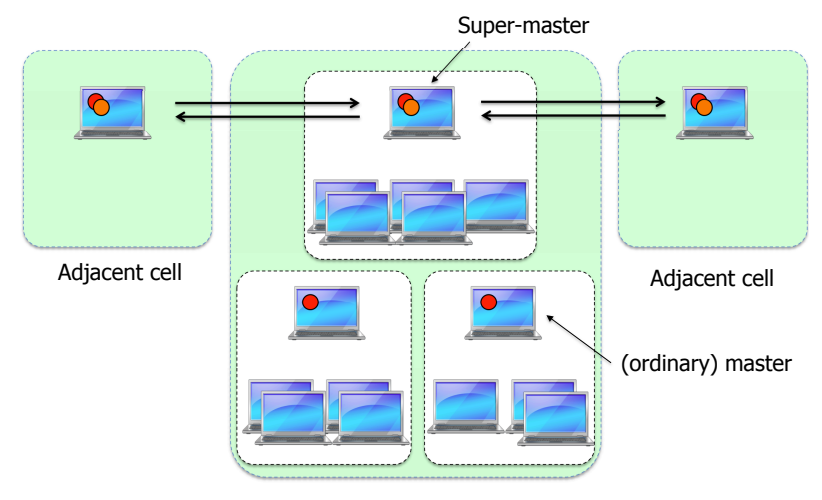

Fig. 6. Proposed hierarchical P2P architecture (ordinary master is marked with red circle and sups-master is marked with orange circle).

Let $M_{i}$ be the set of masters associated with cell $R_{i}$ (note that $M_{i} \neq \emptyset$ as long as $P_{i} \neq \emptyset$ ). In the proposed scheme, we promote arbitrary peer in $M_{i}$ as the super-master and use it as a "contact point" for the communication with adjacent cells, to keep the load of each master sufficiently low. Figure 6 illustrates an overview of the proposed hierarchical $\mathrm{P} 2 \mathrm{P}$ architecture, where ordinary master is marked with red circle and super-master is marked with orange circle. Let $s(u)$ denote the super-master associate with master $u$. The concrete procedure for the communication in the proposed hierarchical architecture is as follows:

Step 1: $u$ periodically notifies the list of current positions of the players in $U(u)$ to super-master $s(u)$. 
Step 2: $s(u)$ combines messages received from the associated masters including itself into a single message and periodically exchanges it with supermasters of adjacent cells (note that $s(u)$ does not "manage" other masters in the same cell, i.e., it simply acts as a contact point to adjacent cells).

Step 3: Upon receiving a combined message from adjacent super-masters, $s(u)$ forwards it to all masters associated with it. With such a scheme, every master can acquire the information on adjacent cells without directly communicating with the corresponding masters.

Step 4: After receiving the combined message, each master $u$ updates the AOI of the players in $U(u)$, and notifies the update to the corresponding players, if necessary. If the number of peers in an adjacent cell exceeds a certain threshold, $u$ conducts such a calculation merely on players which are close to the boundary of the cell associated with $u$.

The effectiveness of the above notification scheme is summarized as follows:

- By delegating the role of communication to the super-master, the load of ordinary masters reduces;

- By combining messages received from several masters into a single message, the load of supermaster also reduces while it causes a delay in the notification to adjacent cells; and

- By limiting the number of players managed by each master by $T_{U}$ and by limiting the number of visible peers in adjacent cells by a constant, the load of masters concerned with AOI management reduces.

Finally, to reduce the load of masters concerned with the management of players across boundaries, the proposed scheme conducts a lazy judgement described as follows. When a player $v$ enters cell $R_{j}$, it selects a master $u$ associated with $R_{j}$ according to the rule described in Section 3.3 and becomes a "tentative" member of subset $U(u)$. It then becomes a "normal" member if it arrives at an inside point of $R_{j}$ at distance $\delta$ from the boundary.

\section{Evaluation}

\subsection{Setup}

The performance of the proposed scheme is evaluated by simulation using PeerSim ${ }^{7}$. In the simulation, we measure the response time of masters concerned with the change of AOI; i.e., Type $\mathrm{C}$ events in Section 3.1. The number of players is fixed in the range from 100 to 700 and the game field is divided into $10 \times 10$ hexagonal cells, where we assume that the game field is topologically equivalent to the surface of a ball. The length of each side of the hexagon is 100 unit length and the AOI of each player is a circle of radius 50 unit length. In a unit time, each peer conducts the following sequential steps:

1. Move to a point in the game field according to the mobility rules described in Section 4.2;

2. Receive (at most) 50 messages in the input buffer;

3. Conduct necessary computation; and

4. Send messages to other players.

More concretely, in Step (4), each peer notifies its current position to all players in its $\mathrm{AOI}$ and the corresponding master. In addition to that, peers promoted as a master or a super-master periodically issues combined messages as was described in Section 3.4. Parameters used in the experiments are summarized in Table 7.

Fig. 7. Parameters.

\begin{tabular}{|c|c|}
\hline Parameter & value \\
\hline Number of players & 100 to 700 \\
Game field & $10 \times 10$ hexagonal cells \\
Cell & Hexagon with side length 100 \\
Area-of-Interest & Circle of radius 50 \\
$\left(T_{U}, T_{L}\right)$ & $(12,5)$ \\
$\alpha$ & $0,50,100$ \\
\hline
\end{tabular}

\subsection{Mobility Patterns}

As for the model of mobility of players, we consider two mobility modes called Random and Straight. 


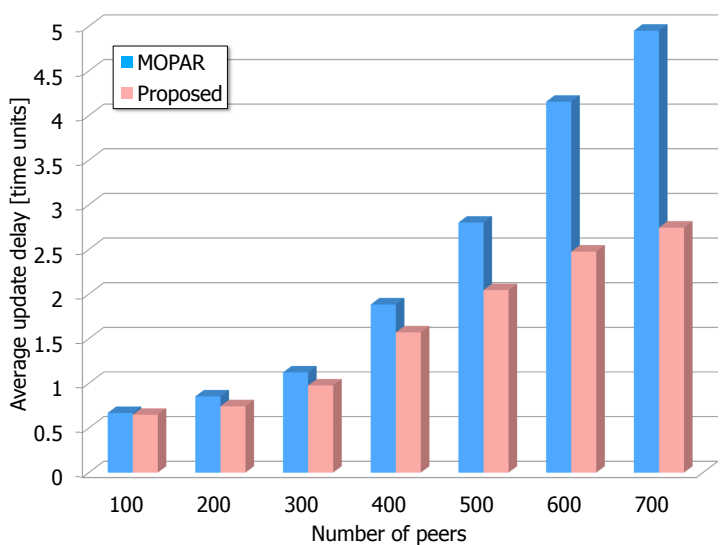

(a) $\alpha=100$ [\%].

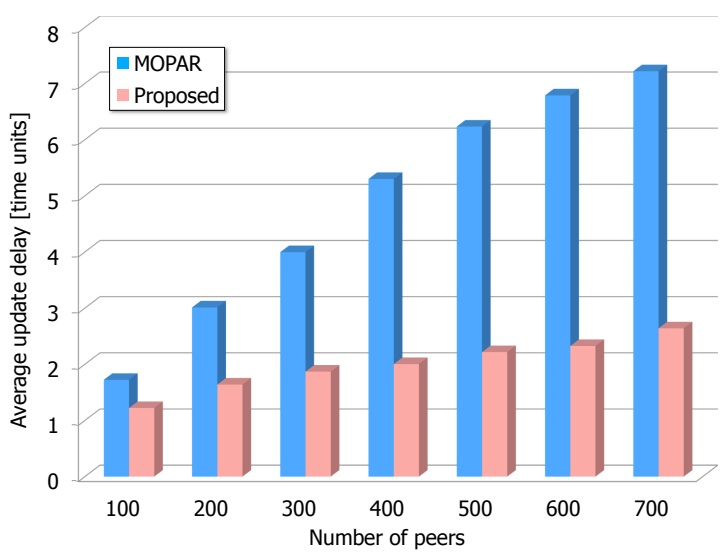

(b) $\alpha=50[\%]$.

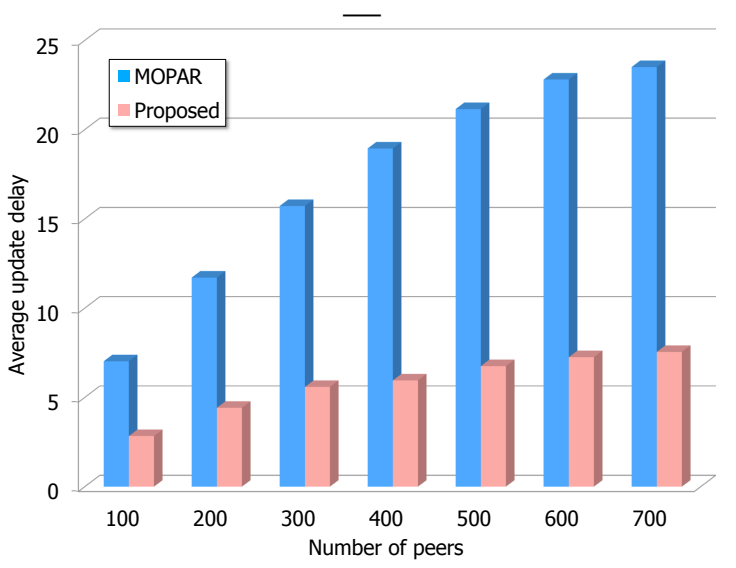

(c) $\alpha=0[\%]$.

Fig. 8. Result of simulations.

In Random, each player conducts random walk. More concretely, in each step, each player follows the movement in the last step with probability $90 \%$ and with probability $10 \%$, it moves to a point at dis- tance at most two [unit length] in a random direction. In Straight, each player moves to a specific cell from the initial (random) position with a speed of at most two [unit length per unit time] and after reaching the target cell, it conduct a random walk within the cell until the end of simulation.

In the experiments, we assume that $\alpha$ [\%] of peers follow Random and $100-\alpha[\%]$ peers follow Straight provided that the initial configuration of the peers is randomly given.

\subsection{Result}

Figure 8 summarizes the simulation results. When $\alpha=100$, the proposed scheme exhibits almost the same performance with MOPAR for small number of peers, since it does not cause the split of subsets. However, as the number of peers increases, it reduces the response time of MOPAR by about $40 \%$ on average. When $\alpha=0$, on the other hand, the proposed scheme significantly improves MOPAR, which is apparently due to the concentration of players to a specific cell. In fact, such a concentration degrades the performance of MOPAR and the split of the subset associated with the target cell effectively reduces the average response time. More specifically, the response time of the proposed scheme is bounded by seven [time units] regardless of the number of peers, and it improves the response time of MOPAR by $70 \%$ when the number of peers is 700. Similar phenomena could be observed for $\alpha=50$. Although the amount of reduction is smaller than the case of $\alpha=0$, it reduces the response time of MOPAR by about $60 \%$ on average.

\section{Concluding Remarks}

In this paper, we propose a threshold-based load balancing scheme for the distributed AOI management in P2P MMPOPGs. The proposed scheme is designed for three-tier P2Ps consisting of ordinary peers, masters and super-masters, and the split of a subset of peers is conducted by solving the graph bipartition problem using KL algorithm. Simulation results indicate that the proposed load balancing scheme reduces the response time of MOPAR by about $50 \%$. 
A future work is to conduct extensive simulations to evaluate: 1) the continuity of the change of AOI due to split-and-merge operations; 2) the impact of two thresholds to the performance; and 3) the response time of the proposed scheme in actual distributed environment. Another issue is to enhance the churn tolerance of the proposed scheme.

\section{References}

1. E. Carlini, L. Ricci and M. Coppola. "Reducing server load in MMOG via P2P gossip." In Proc. 11th NetGames, 2012, pages 1-2.

2. S.-Y. Hu, J.-F. Chen and T.-H. Chen. "VON: A Scalable Network for Virtual Environments," IEEE Network, 20(4): 22-31, 2006.

3. T. Iimura, H. Hazeyama and Y. Kadobayashi. "Zoned federation of game servers: A peer-to-peer approach to scalable multi-player online games." In Proc. the 3rd ACM NetGames, 2004, pages 116-120.

4. B. W. Kernighan and S. Lin. "An efficient heuristic procedure for partitioning graphs." Bell Systems Technical Journal, 49(2): 291-307, 1970.
5. B. Knutsson, H. Lu, W. Xu and B. Hopkins. "Peerto-peer support for massively multiplayer games." In Proc. IEEE INFOCOM, Vol. 1, 2004.

6. M. Merabti and A. El Rhalibi. "Peer-to-peer architecture and protocol for a massively multiplayer online game.” In Proc. IEEE GlobeCom Workshops, 2004, pages 519-528.

7. A. Montresor and M. Jelasity. "Peersim: A Scalable P2P simulator." In Proc. of the 9th Int. Conf. on Peerto-Peer (P2P' 09), 2009, pages 99-100.

8. A. El Rhalibi and M. Merabiti. "Interest management and scalability issues in P2P MMOG." In Proc. the 3rd IEEE Consumer Communications and Networking Conf., 2006, pages 1188-1192.

9. A. Rowstron and P. Druschel. "Pastry: Scalable, distributed object location and routing for large scale peer-to-peer systems." In Proc. of IFIP/ACM Middleware, 2001, pages 329-350.

10. A. Yu and S. T. Vuong. "MOPAR: A mobile peer-topeer overlay architecture for interest management of massively multiplayer online games," Proc. of NOSS$D A V, 2005$, pages 99-104.

11. A. Yu and S. T. Vuong. "A DHT-based hierarchical overlay for Peer-to-Peer MMOGs over MANETs." In Proc. 7th IWCMC, 2011, pages 1475-1480. 